\title{
Reflets
}

Revue ontaroise d'intervention sociale et communautaire

\section{Sondage auprès des diplômées et diplômés de l'École de service social de l'Université Laurentienne}

\section{Richard Carrière, Carol Kauppi et Herb Nabigon}

Volume 7, numéro 2, automne 2001

Le travail social en Ontario

URI : https://id.erudit.org/iderudit/026366ar

DOI : https://doi.org/10.7202/026366ar

Aller au sommaire du numéro

Éditeur(s)

Reflets : Revue ontaroise d'intervention sociale et communautaire

ISSN

1203-4576 (imprimé)

1712-8498 (numérique)

Découvrir la revue

Citer cet article

Carrière, R., Kauppi, C. \& Nabigon, H. (2001). Sondage auprès des diplômées et diplômés de l’École de service social de l'Université Laurentienne. Reflets, 7(2), 192-204. https://doi.org/10.7202/026366ar

Tous droits réservés (C) Reflets : Revue ontaroise d'intervention sociale et communautaire, 2001
Ce document est protégé par la loi sur le droit d'auteur. L'utilisation des services d'Érudit (y compris la reproduction) est assujettie à sa politique d'utilisation que vous pouvez consulter en ligne.

https://apropos.erudit.org/fr/usagers/politique-dutilisation/ 


\section{Sondage auprès des diplômées et diplômés de l'École de service social de 1'Université Laurentienne}

par

Richard Carrière, Carol Kauppi et Herb Nabigon, École de service social, Université Laurentienne

\section{Introduction}

Dans le cadre du rapport d'auto-évaluation en vue de l'agrément par l'Association canadienne des Écoles de service social, nous avons mené un sondage auprès des diplômées et diplômés des trois composantes de l'École de service social de l'Université Laurentienne. Il est important de souligner que celle-ci est la seule école canadienne à offrir un programme à trois groupes distincts d'étudiantes et d'étudiants, afin de répondre aux besoins linguistiques et culturels de la population du Nord-est de l'Ontario.Ainsi,l'École offre son programme de baccalauréat à une population estudiantine anglophone, francophone et amérindienne et chaque composante a son propre corps professoral. Bien que les trois composantes offrent sensiblement les mêmes cours de base, chacune d'elles ajoute des éléments qui correspondent à sa propre réalité.Ainsi les résultats du sondage présentés ci-dessous sont organisés de telle sorte qu'ils refléteront les réponses distinctes des trois composantes.

Le sondage, mené en janvier 2001, visait deux objectifs. Dans un premier temps, nous cherchions à établir un profil de nos 
finissants et finissantes en posant un regard particulier sur leur expérience de travail. Dans un deuxième temps, nous voulions connaitre leur satisfaction par rapport à leur programme d'étude en service social.

Afin d'assurer le plus haut taux possible de réponses, nous avons choisi de faire un sondage par téléphone plutôt que par courrier. Ce sondage a été effectué par une adjointe de recherche pendant le mois de janvier 2001, à partir d'une liste de 440 étudiantes ayant gradué durant la période s'étendant de 1986 à 2000. Cette liste provient du Bureau des anciens de l'Université Laurentienne. Bien que le sondage par téléphone se soit avéré un moyen approprié d'enquête lorsque nous réussissions à rejoindre les diplômées et diplômés, il faut dire qu'en raison de la pauvreté des informations contenues dans cette liste (mauvais numéro de téléphone, changement d'adresse, par ex.), nous n'avons pas pu contacter en un grand nombre. Néanmoins, nous avons réussi à interviewer 105 d'entre elles: 60 de la composante anglophone (BSW), 26 de la composante francophone (BSS) et 19 de la composante amérindienne (NHS).

\section{Caractéristiques des répondantes et des répondants}

Comme c'est le cas à l'échelle nationale dans la profession du service social, les femmes composent la grande partie de nos diplômées; $87 \%$ sont des femmes et $13 \%$ sont des hommes. D'ailleurs, la proportion de femmes est plus élevée que la proportion nationale où $71,5 \%$ des personnes œuvrant dans le domaine du service social sont des femmes (ACESS, 2001).

L'âge moyen de nos diplômées et diplômés était de 36 ans. En examinant l'âge par composante d'étude, on constate que la moyenne d'âge était plus élevée chez les diplômées et diplômés de la composante amérindienne (40 ans) tandis que celles et ceux de la composante francophone étaient plus jeunes (33 ans). 
La figure 1 présente la distribution des finissantes et des finissants qui ont complété leur programme en prenant leurs cours sur campus, à distance ou une combinaison de ces deux modes d'étude. Les diplômées et diplômés de la composante amérindienne ont eu recours davantage aux cours à distance $(16 \%)$ que celles et ceux du BSW, alors qu'une plus grande proportion de diplômées et diplômés francophones (BSS) avaient combiné les cours à distance et sur campus (35\%). Comme le programme anglophone (BSW) n'offre que très peu de ses cours sous le format distance, il n'est pas surprenant de constater le peu d'étudiantes et d'étudiants ayant suivi des cours à distance. À ce sujet, il faut dire que les finissantes et finissants anglophones n'ont pas choisi de suivre davantage des cours optionnels à distance dans les domaines des arts et des humanités, malgré l'éventail important de cours à distance offerts à l'Université Laurentienne.

Figure 1: Distribution des diplômées par programme et par modes d'étude

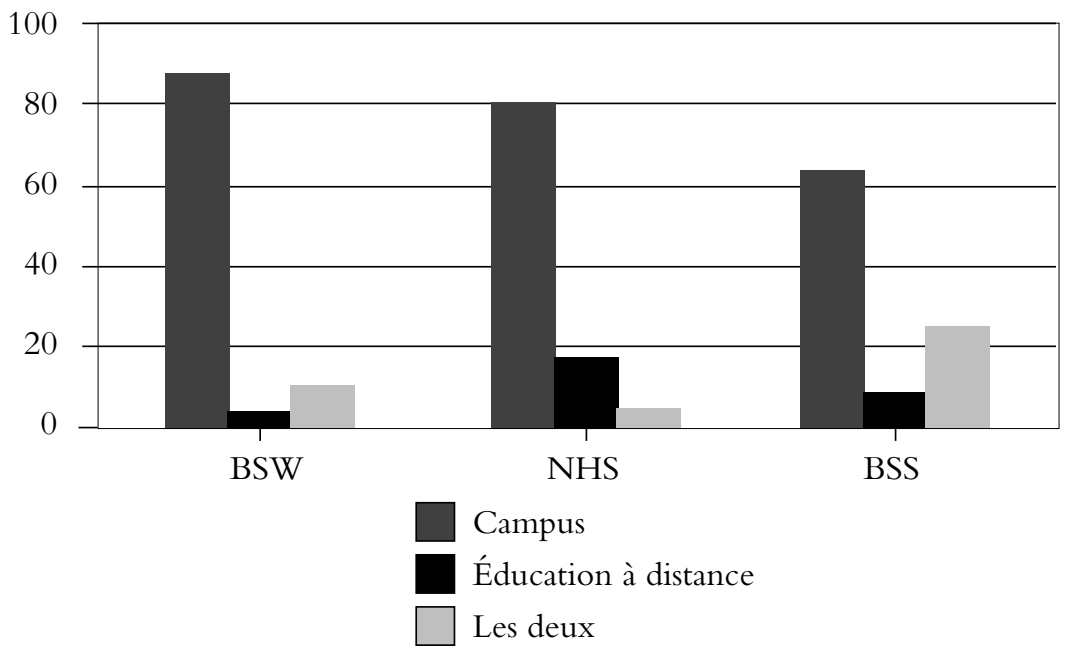

La majorité des diplômées et diplômés est originaire de Sudbury ou de d'autres communautés du Nord $(78 \%)$, les autres proviennent du Sud (16\%) ou de l'Est (6\%) de l'Ontario. 
L'emplacement géographique de l'École de service social à Sudbury a été le principal facteur de décision des diplômées et diplômés de la composante anglophone et francophone dans leur choix du programme d'étude (BSW: $43 \%$; BSS: $42 \%$ ). Par ailleurs, la disponibilité d'un programme dans leur langue ou leur culture a été le premier facteur mentionné par $58 \%$ des diplômées et diplômés de la composante NHS. La dimension linguistique et culturelle du programme s'est aussi révélée un facteur déterminant pour $31 \%$ des diplômées et diplômés francophones.

En 2001, près des deux tiers des finissantes et finissants, peu importe la composante d'appartenance, demeuraient toujours à Sudbury ou dans les communautés du Nord $(64 \%)$ tandis qu'un cinquième d'entre eux habitaient au Sud (22\%) et une plus petite proportion (5\%) habitaient l'Est de la province ontarienne (tableau 1). Quelques diplômées et diplômés de chacune des composantes avaient migré hors de la province $(9 \%)$. Ces diplômées et diplômés habitaient et travaillaient dans des endroits tels le Québec et la ColombieBritannique.

Tableau 1: Nombre de diplômées et diplômés par composante et par résidence avant et après leur graduation

\begin{tabular}{|l|c|c|c|c|c|c|c|c|}
\hline \multicolumn{4}{|c|}{ Lieu de résidence avant les études } & \multicolumn{3}{|c|}{ Lieu de résidence après les études } \\
\hline & $\begin{array}{c}\text { Sudbury } \\
\text { et Nord }\end{array}$ & Sud & Est & $\begin{array}{c}\text { Hors } \\
\text { province }\end{array}$ & $\begin{array}{c}\text { Sudbury } \\
\text { et Nord }\end{array}$ & Sud & Est & $\begin{array}{c}\text { Hors } \\
\text { province }\end{array}$ \\
\hline \hline BSW & 45 & 10 & 5 & - & 38 & 14 & 4 & 4 \\
\hline NHS & 12 & 5 & 1 & 1 & 12 & 4 & - & 3 \\
\hline BSS & 26 & 2 & 1 & - & 17 & 5 & 2 & 2 \\
\hline Total & 83 & 17 & 7 & 1 & 67 & 23 & 6 & 9 \\
\hline
\end{tabular}

N: 105 (Sud: Sud de l'Ontario; Est: Est de l'Ontario) 


\section{Expérience éducative à l'École de service social}

\section{Niveau de satisfaction avec divers éléments du programme d'étude}

Le tableau 2 montre les niveaux de satisfaction des diplômées et des diplômés par rapport à leurs études à l'École de service social à l'Université Laurentienne. En général, on manifeste un niveau de satisfaction élevé par rapport au programme d'étude. Le plus haut taux de satisfaction porte sur les horaires et le plus faible est lié à la qualité des salles de classe. Dans l'ensemble, seulement $4 \%$ des répondantes et répondants ont exprimé leurs insatisfactions avec les divers éléments du programme. Les éléments du programme qui ont provoqué le plus de mécontentement était la qualité des salles de classe pour la composante amérindienne et la pénurie de choix de cours pour la clientèle anglophone.

Le taux de satisfaction par rapport à l'ensemble du programme d'étude est plus élevé dans la composante amérindienne (95\% des répondantes et répondants) que chez la composante anglophone (68\% des répondantes et répondants). Quant aux diplômées et diplômés de la composante francophone, $81 \%$ expriment un niveau de satisfaction élevé pour l'ensemble du programme. Le taux de satisfaction peu élevé des diplômées et diplômés anglophones est peut-être la conséquence de la pénurie du choix de cours disponibles relevée précédemment. 
Tableau 2: Niveau de satisfaction sur les divers éléments du programme par composante

\begin{tabular}{|c|c|c|c|c|c|c|c|c|c|}
\hline Aspects & \multicolumn{3}{|c|}{$\%$ Très insatisfait } & \multicolumn{3}{|c|}{$\%$ Très satisfait } & \multicolumn{3}{|c|}{ Score moyen } \\
\hline \multirow[b]{2}{*}{$\begin{array}{l}\text { Le programme } \\
\text { (en général) }\end{array}$} & BSW & NHS & BSS & BSW & NHS & BSS & BSW & NHS & BSS \\
\hline & 5,0 & 5,3 & - & 68,4 & 94,7 & 80,7 & 6,77 & 7,74 & 7,27 \\
\hline $\begin{array}{l}\text { Qualité de } \\
\text { l'enseignement }\end{array}$ & 6,7 & 5,3 & - & 63,3 & 89,5 & 65,4 & 6,67 & 7,53 & 6,77 \\
\hline Choix de cours & 10,0 & 5,3 & 3,8 & 41,6 & 89,5 & 65,4 & 5,83 & 7,37 & 6,81 \\
\hline Contenu des cours & 3,3 & - & 3,8 & 65,0 & 100 & 61,5 & 6,70 & 7,89 & 6,81 \\
\hline Horaire des cours & 1,7 & 5,3 & - & 71,6 & 79,0 & 88,0 & 7,02 & 7,37 & 7,76 \\
\hline $\begin{array}{l}\text { Nombre de cours } \\
\text { suivis }\end{array}$ & 5,0 & 一 & 3,8 & 73,3 & 84,3 & 77,0 & 7,00 & 7,58 & 7,12 \\
\hline Travaux requis & 5,0 & 5,3 & 3,8 & 73,4 & 89,5 & 80,8 & 6,90 & 7,68 & 7,27 \\
\hline $\begin{array}{l}\text { Qualité des salles } \\
\text { de classe }\end{array}$ & 5,0 & 11,8 & 4,0 & 58,3 & 64,6 & 56,0 & 6,77 & 6,59 & 6,84 \\
\hline
\end{tabular}

\section{Niveaux de satisfaction liés au stage et à ses divers éléments}

En général, les diplômées et diplômés sont très satisfaits de leurs expériences de stage (Tableau 3). Une majorité indique un niveau élevé de satisfaction sur la durée des stages, la supervision offerte par l'agence et celle offerte par la consultation académique. Par ailleurs, les diplômées et diplômés expriment un niveau de satisfaction moins élevé par rapport au processus du choix des stages. Ce sont les diplômées et diplômés francophones qui expriment le plus faible taux de satisfaction (46\%). Inversement, une forte majorité des répondantes et répondants amérindiens (89\%) exprime un niveau de satisfaction élevé quant à leur choix 
de stage, tandis que 60 \% des diplômées et diplômés anglophones font de même.

Tableau 3: Niveaux de satisfaction en matière de stages pratiques selon leurs divers éléments

\begin{tabular}{|l|c|c|c||c|c|c|c|c|c|}
\hline Aspects & \multicolumn{3}{|c|}{ \% Très insatisfait } & \multicolumn{3}{c|}{ \% Très satisfait } & \multicolumn{3}{c|}{ Score moyen } \\
\hline \multirow{2}{*}{$\begin{array}{l}\text { Field placements } \\
\text { (overall) }\end{array}$} & BSW & NHS & BSS & BSW & NHS & BSS & BSW & NHS & BSS \\
\cline { 2 - 11 } & 5,1 & - & 7,7 & 69,9 & 94,7 & 61,5 & 7,2 & 8,0 & 6,9 \\
\hline $\begin{array}{l}\text { Duration of } \\
\text { placement }\end{array}$ & - & - & 3,8 & 84,5 & 63,1 & 92,3 & 7,5 & 7,6 & 7,5 \\
\hline Choice of placements & 14,0 & 5,6 & 15,0 & 69,7 & 88,9 & 46,1 & 6,6 & 7,6 & 5,8 \\
\hline Agency supervision & 3,5 & - & 12,0 & 68,4 & 89,4 & 69,2 & 7,0 & 7,8 & 6,8 \\
\hline Faculty supervision & 6,9 & - & 7,7 & 60,3 & 94,7 & 61,5 & 6,7 & 7,8 & 6,5 \\
\hline $\begin{array}{l}\text { Process of choosing } \\
\text { placements }\end{array}$ & 16,0 & 5,9 & 24,0 & 35,1 & 88,2 & 48,0 & 5,7 & 7,5 & 5,7 \\
\hline $\begin{array}{l}\text { N=105 (Les étudiantes et étudiants ont évalué ces aspects sur une échelle de 9 points, } 1 \\
\text { étant le niveau le moins élevé et, 9 le plus élevé. Dans ce tableau, le niveau le plus élevé } \\
\text { reflète une combinaison des scores allant de 7 à 9) }\end{array}$ \\
\hline
\end{tabular}

\section{Satisfaction concernant les stages : avant et après 1996}

Les compressions budgétaires et la restructuration du secteur des services sociaux depuis 1995 ont pu contribuer à l'expérience de stages des diplômées. Par exemple, après 1995, il est devenu plus difficile, sinon impossible, de respecter les choix des étudiantes et étudiants par rapport à certains lieux de stage. En effet, les réductions de fonds et les pressions accrues qui en découlent ont eu leurs effets sur le personnel des agences et sur leur disponibilité de supervision. Ainsi, plusieurs agences ont arrêté d'accepter des stagiaires, réduisant d'autant les choix possibles. La figure 2 montre que la satisfaction globale, par rapport aux stages, a diminué légèrement parmi les diplômées et diplômés du BSW et du BSS 
ayant terminé leurs études après 1995. Les figures 2.1 et 2.3 montrent que la baisse du niveau de satisfaction s'est fait sentir surtout en relation avec les éléments du choix de stages et le processus du choix de stages. Au cours de la dernière année, certaines agences préférées par les étudiantes, telles la Société d'aide à l'enfance de Sudbury, ont de nouveau ouvert leurs portes aux stagiaires, permettant ainsi un certain redressement de la situation et de taire le mécontentement qui commençait à poindre.

Figure 2: Niveau de satisfaction avec les stages avant et après 1996 par composante

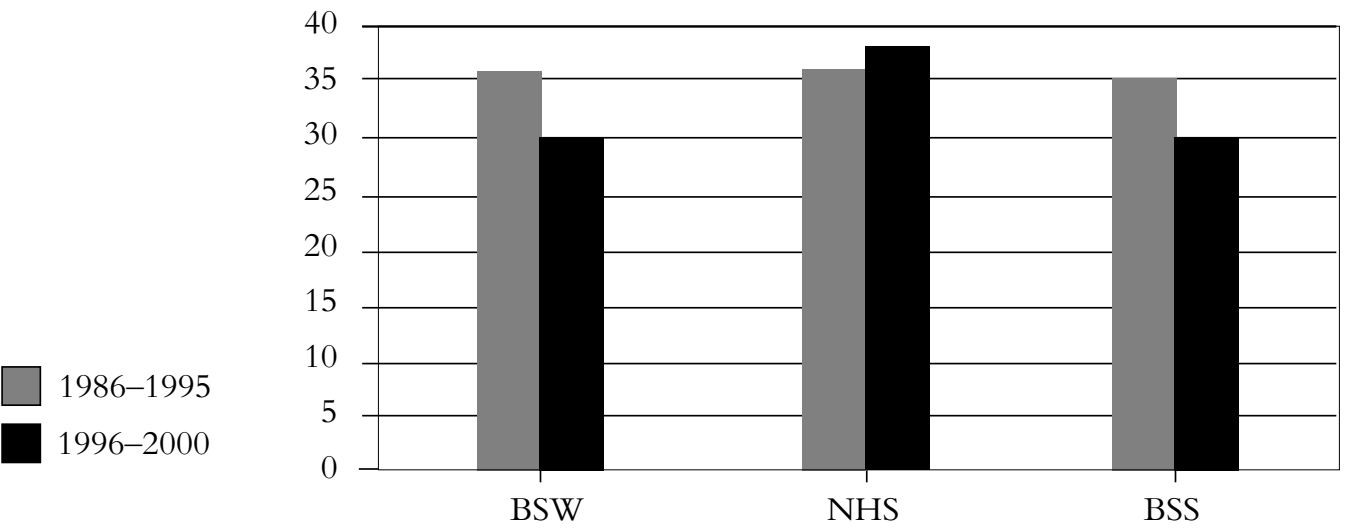

Figure 2.1: Niveau de satisfaction par rapport au choix de stages avant et après 1996 par composante.

1986-1995

1996-2000

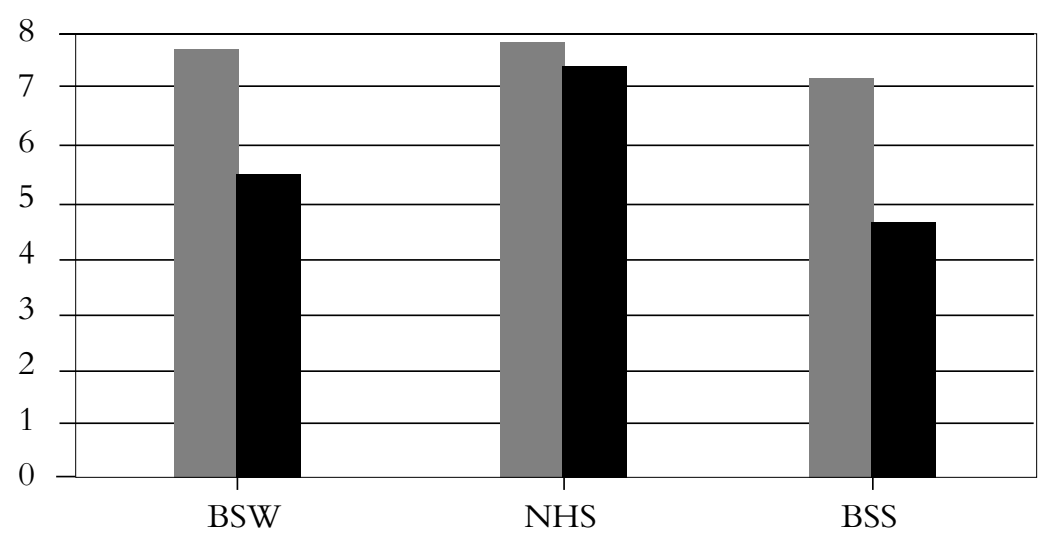


Figure 2.2: Niveau de satisfaction avec la supervision par l'agence avant et après 1996 par composante.

1986-1995

1996-2000

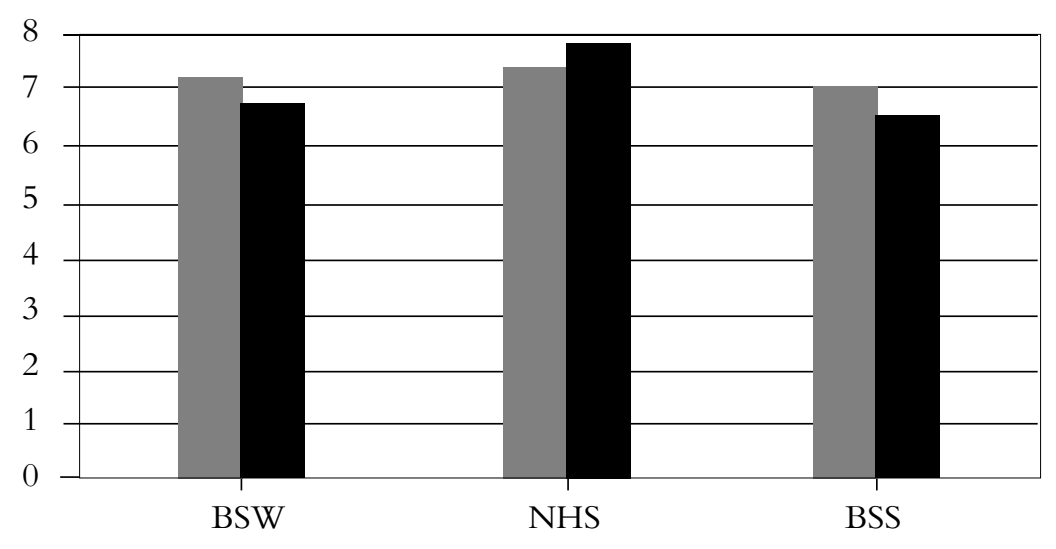

Figure 2.3: Niveau de satisfaction avec le processus de choisir les stages avant et après 1996 par composante

1986-1995

1996-2000

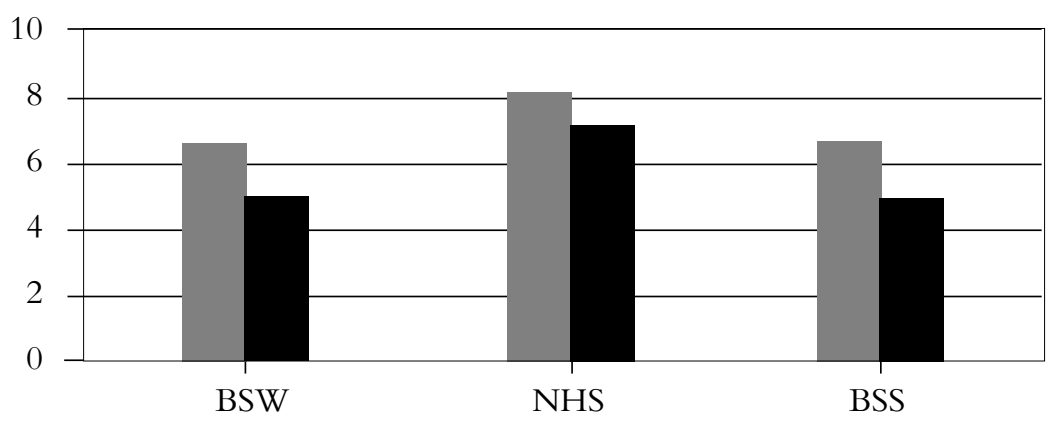

\section{Préparation à la pratique}

En plus de compiler de l'information sur le niveau de satisfaction par rapport au programme d'étude et l'appréciation des stages, les diplômées et diplômés ont aussi été invitées à faire part de leur perception concernant la préparation au marché du travail offerte par leur étude en service social. La majorité des diplômées et diplômés des trois composantes ont affirmé que leur formation était adéquate (NHS - $94 \%$, BSW - $70 \%$ et BSS - 59\%) et 
les avaient bien préparés à occuper leur emploi. Celles et ceux ayant indiqué que leurs études ne les avaient pas préparés adéquatement, ont souligné le caractère généraliste du programme. Ces diplômées et diplômés ne sentaient pas que leur préparation était assez spécifique pour répondre aux exigences de leur champ de travail.

\section{Désir de poursuivre des études supérieures}

Les diplômées et diplômés ont été interrogées sur leur désir de poursuivre des études supérieures. Bien qu'une faible majorité des diplômées anglophones (53\%) aient exprimé le désir de poursuivre des études supérieures, seulement $42 \%$ des diplômées amérindiennes et $27 \%$ des diplômées francophones ont mentionné un tel intérêt.

\section{Portrait de la situation en emploi}

Une forte majorité des diplômées et des diplômés était sur le marché du travail au moment du sondage, $6 \%$ étant sans emploi. Ces données sont similaires à celles qui sont présentées dans le rapport Profile of Recent Graduates, publié par Développement des ressources humaines Canada (2000b). Celui-ci montre que $93 \%$ des diplômées en service social sont sur le marché du travail deux ans après leurs études universitaires. Une forte proportion des diplômées et diplômés de l'École de service social de l'Université Laurentienne, soit $88 \%$, travaille à temps plein, ce qui est supérieur aux $83 \%$ des données nationales (HRDC 2000b). Plus de la moitié des diplômées et diplômés de l'École de service social qui ne travaillaient pas au moment de notre sondage poursuivaient des études supérieures (7 sur 13). Deux autres individus étaient en congé de maladie ou en congé de maternité. 
Tableau 4: Nombre de répondantes et répondants par emploi en service social (BSW-NHS-BSS)

\begin{tabular}{|l|c|c|c|c|}
\hline Domaines d'emploi & BSW & NHS & BSS & Total \\
\hline Société d'aide à l'enfance & 12 & 3 & 4 & 20 \\
\hline Services de santé & 10 & 1 & 4 & 15 \\
\hline Services à l'enfance et la famille & 7 & 3 & 4 & 13 \\
\hline Écoles de service social & 4 & 1 & 5 & 10 \\
\hline Services auprès des Amérindiens & - & 5 & - & 5 \\
\hline Services correctionnels & 2 & 2 & - & 4 \\
\hline Services auprès des femmes & 2 & 2 & - & 4 \\
\hline Services d'aide sociale & 4 & - & - & 4 \\
\hline Services de réhabilitation & 4 & - & - & 4 \\
\hline Pratique privée & 2 & - & - & 2 \\
\hline Autres & 2 & 1 & 3 & 6 \\
\hline Total & $\mathbf{4 9}$ & $\mathbf{1 8}$ & $\mathbf{2 0}$ & $\mathbf{8 7}$ \\
\hline Note : 18 répondantes et répondant n'ont pas indiqué leur emploi. \\
\hline
\end{tabular}

\section{Revenu}

L'analyse du revenu annuel des diplômées et diplômés révèle que $57 \%$ gagnent plus de 40000 \$ par année. Ces données suggèrent que la majorité gagne un salaire comparable ou supérieur aux diplômées et diplômés canadiens en service social. Selon HRDC, le salaire moyen, deux ans après la fin des études, des travailleuses sociales et des travailleurs sociaux détenant un baccalauréat, était $34500 \$$, celles et ceux détenant une maîtrise gagnaient, en moyenne, $40400 \$$. Parmi les répondantes et les répondants des composantes amérindienne, anglophone et francophone, $61 \%$, $57 \%$ et $52 \%$ avaient respectivement un revenu annuel supérieur à 40000 \$ (voir Figure 3). 
Figure 3: Revenu annuel par composante (BSW-NHS-BSS)

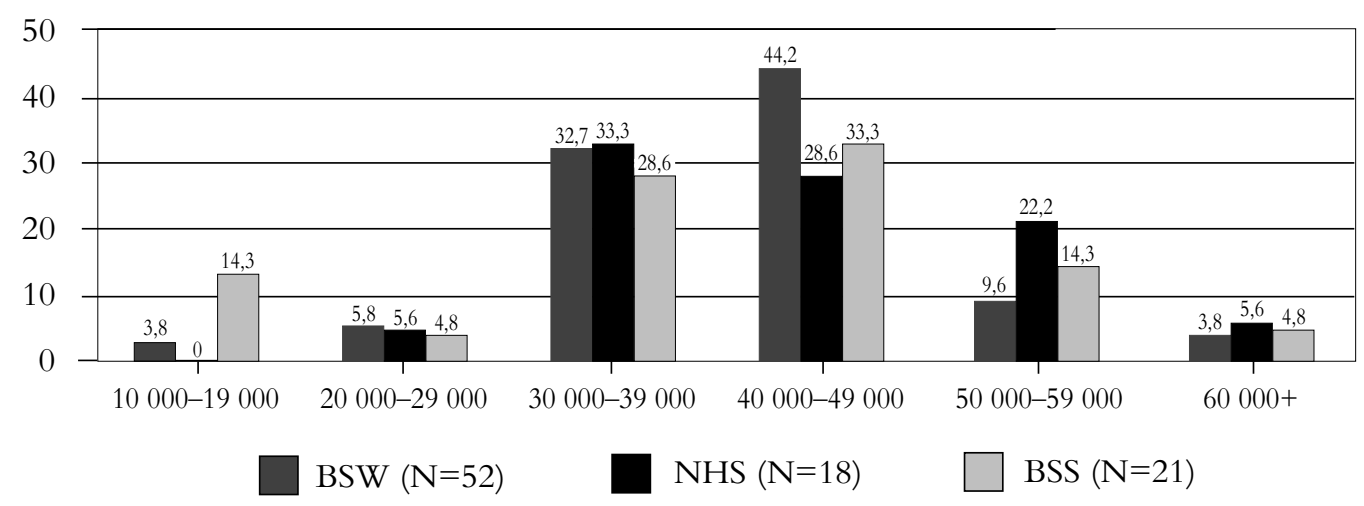

\section{Affiliation professionnelle}

Même si une forte majorité des diplômées et diplômés de l'École de service social de l'Université Laurentienne travaille dans le domaine du service social, la plupart ne sont pas membres de l'Association des travailleuses et des travailleurs sociaux de l'Ontario (ATTSO), ni de l'Ordre des travailleurs sociaux et des techniciens en service social (OTSTSS). La figure 4 montre les affiliations professionnelles des diplômées et diplômés. Les résultats suggèrent qu'une forte proportion d'anglophones ont choisi de devenir membres de l'ATTSO et de l'OTSTSS comparativement aux personnes d'origine amérindienne et francophone.

Figure 4:Taux de répondantes et répondants oeuvrant en service social par leurs affiliations professionnelles et par composante.

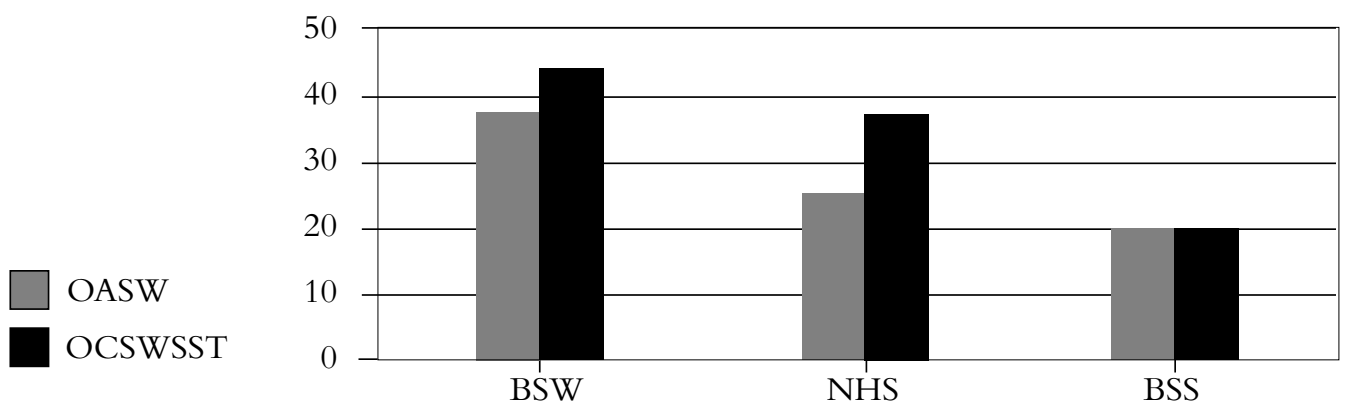




\section{Conclusion}

Les données de ce premier sondage des diplômées et diplômés de l'École de service social de l'Université Laurentienne, selon ses trois composantes, permettent de constater le haut niveau de satisfaction de celles et ceux qui ont suivi ce programme. De plus, leur formation leur a permis d'obtenir un emploi à temps plein en service social. Mission accomplie! En examinant les éléments plus faibles, nous seront en mesure d'établir les stratégies capables d'améliorer notre programme unique et ses dimensions spécifiques liées aux trois composantes. Nous sommes fiers de nos diplômées et diplômés. Nous leur souhaitons un franc succès dans leur carrière.

\section{Bibliographie}

Association canadienne des Écoles de service social (ACESS) (2001). Le travail social au Canada: une profession essentielle, Ottawa, ACESS.

Human Resources Development Canada (2000a). Job Futures 2000, http://www11.hrdc-drch/ jf2 comp.compare

Human Resources Development Canada (2000b).Job Futures 2000, http://www11.hrdc-drhc.gc.ca/ jobfutures/fos/U890.html

\section{Note}

1. BSS: baccalauréat en service social;BSW:Bachelor of social Work; NHS: Native Human Services. 